\title{
CONSTRUCTION OF OPTIMAL GRID INTERPOLATION FORMULAS IN SOBOLEV SPACE $\widetilde{L_{2}^{m}}(H)$ OF PERIODIC FUNCTION OF $n$ VARIABLES BY SOBOLEV METHOD
}

\author{
N.H. MAMATOVA, A.R. HAYOTOV, KH.M. SHADIMETOV
}

\begin{abstract}
In the present work we consider the problem of constructing optimal grid interpolation formulas in the space $\widetilde{L_{2}^{m}}(H)$ of periodic function of $n$ variables. We find the coefficients of grid interpolation formulas.
\end{abstract}

Keywords: Sobolev space, optimal interpolation formula, properties of the discrete analogue of the operator $\Delta^{m}$, optimal coefficients.

\section{MAIN RESUlts}

Let us remind the definition of Sobolev space $\widetilde{L_{2}^{(m)}}(H)$ of periodic functions of $n$ variables. Let a function $\varphi(x)$ be periodic with the periods matrix $H$,

$$
\varphi(x+H \gamma)=\varphi(x), \quad x \in \mathbb{R}^{n},
$$

where $\gamma$ is an arbitrary integer column vector, $H$ is a matrix of size $n \times n$ having unit determinant.

To the matrix $H$ we associate its fundamental parallelepiped $\Omega_{0}$ letting

$$
\Omega_{0}=\left\{x \in \mathbb{R}^{n}: \quad x=H y, \quad 0 \leqslant y_{j}<1, \quad j=1,2, \ldots, n\right\} .
$$

Suppose $2 m>n$ and the integral

$$
\int_{\Omega_{0}} \sum_{|\alpha|=m} \frac{m !}{\alpha !}\left(D^{\alpha} \varphi(x)\right)^{2} d x
$$

is finite, where $\alpha$ is a multi-index, $\alpha=\left(\alpha_{1}, \alpha_{2}, \ldots, \alpha_{n}\right), \alpha !=\alpha_{1} ! \alpha_{2} ! \ldots \alpha_{n} !,|\alpha|=\sum_{j=1}^{n} \alpha_{j}$, $D^{\alpha} \varphi(x)=\frac{\partial^{|\alpha|} \varphi(x)}{\partial x_{1}^{\alpha_{1}} \partial x_{2}^{\alpha_{2} \ldots \partial x_{n}^{\alpha}}}$.

The norm in $L_{2}^{(m)}(H)$ is defined by the formula

$$
\left\|\varphi(x) \mid \widetilde{L_{2}^{(m)}}(H)\right\|=\left[\int_{\Omega_{0}} \sum_{|\alpha|=m} \frac{m !}{\alpha !}\left(D^{\alpha} \varphi(x)\right)^{2} d x\right]^{\frac{1}{2}} .
$$

As the elements of the spaces $\widetilde{L_{2}^{(m)}}(H)$ serve the functions differing by a constant.

The space $\widetilde{L_{2}^{(m) *}}(H)$ comprises of all periodic functionals orthogonal to one, i.e.,

$$
(\ell(x), 1)=0 .
$$

N.H. Mamatova, A.R. Hayotov, Kh.M. Shadimetov, Construction of optimal grid interpolaTION FORMULAS IN SOBOLEV SPACE $\widetilde{L_{2}^{m}}(H)$ OF PERIODIC FUNCTION OF $n$ VARIABleS By SOBOlEV METHOD.

(C) Mamatova N.H., Hayotov A.R., Shadimetov Kh.M. 2013.

Submitted December 20, 2011. 
Consider the interpolation formula

$$
\varphi(x) \cong P_{\varphi}(x)=\sum_{k=1}^{N} C_{k}(x) \varphi\left(x^{(k)}\right)
$$

in the space $\widetilde{L_{2}^{(m)}}(H)$, the points $x^{(k)} \in \Omega_{0}$ and parameters $C_{k}(x)$ are called respectively nodes and coefficients of interpolation formula $(2)$.

One of the main problems of interpolation theory is to find the maximum of the error of the interpolation formula $\varphi(x) \cong P_{\varphi}(x)$ in functional spaces. The error at a point $z$ is the value of an error functional on the function $\varphi$,

$$
\begin{gathered}
(\ell(x), \varphi) \equiv \varphi(z)-P_{\varphi}(z)=\varphi(z)-\sum_{k=1}^{N} C_{k}(z) \varphi\left(x^{(k)}\right)= \\
\left.=\int_{\Omega_{0}}\left[\left(\delta(x-z)-\sum_{k=1}^{N} C_{k}(z) \delta\left(x-x^{(k)}\right)\right) * \phi_{0}\left(H^{-1} x\right)\right] \varphi(x) d x, 1\right]
\end{gathered}
$$

where $\delta(x)$ is the Dirac delta-function, $\phi_{0}\left(H^{-1} x\right)=\sum_{\beta} \delta(x-H \beta)$,

$$
\ell(x)=\left(\delta(x-z)-\sum_{k=1}^{N} C_{k}(z) \delta\left(x-x^{(k)}\right)\right) * \phi_{0}\left(H^{-1} x\right)
$$

is the error functional of the interpolation formula.

The variable parameters of the interpolation formula are the nodes $x^{(k)}$ and the coefficients $C_{k}(z)$. An optimal interpolation formula is that whose error functional has the minimal norm in $\widetilde{L_{2}^{(m) *}}(H)$ w.r.t. variations of the coefficients under given nodes. If the nodes $x^{(k)}$ are the points of a grid, i.e., are located at the points $x^{(\gamma)}=h H \gamma$, the interpolation formula is called a grid one. Here $h$ is a small parameter called grid spacing.

In the present work we construct optimal grid interpolation formulas in the Sobolev space $\widetilde{L_{2}^{(m)}}(H)$. A similar problem was first formulated and considered by Sobolev [1], where there was found an extremal function of the interpolation formula in the space $W_{2}^{(m)}$ of functions whose derivatives of order $m$ are square integrable.

It should be noted that the solution for $p=2$ (Holladay theorem [2]) to the problem on minimization of $L_{p}$-norm of $m$ th derivative of the function interpolating given values $y_{i}$ at the points $x_{i}$ led to the developing of spline theory. Later this problem was studied in numerous works in a more general formulation as the problem of minimization of a functional under restrictions (see, for instance, [3-8]).

The main result of the present work is

Theorem. In the Sobolev space $\widetilde{L_{2}^{(m)}}(H)$ there exists the unique optimal grid interpolation formula (2) with error functional (3). Its coefficients are determined by the formula

$$
\stackrel{\circ}{C}([\beta] ; z)=h^{n}\left(1+\sum_{\gamma \neq 0} \frac{\exp \left(2 \pi i H^{-1}\left(H h \beta^{*}-z\right) \gamma\right)}{\left|H^{-1 *} \gamma\right|^{2 m}} \cdot K(\gamma)\right),
$$

where $K(\gamma)=\left[\sum_{\substack{t \\ t \neq h \gamma}} \frac{1}{\left|H^{-1 *}\left(h^{-1} t-\gamma\right)\right|^{2 m}}\right]^{-1}$.

\footnotetext{
${ }^{1}$ The intergal of Dirac function is a historical convention, $\int \delta(x-z) \varphi(x) d x \equiv\left(\delta_{z}, \varphi\right) \equiv \varphi(z)$; the convolution of $\delta$-functions is defined as $\delta(x-a) \delta(x-b)=\delta(x-(a+b))$.
} 


\section{EXTREMAL FUNCTION OF INTERPOLATION FORMULA}

In order to find explicitly the norm of the error functional $\ell(x)$ in the space $\widetilde{L_{2}^{(m)}}(H)$ we shall employ the notion of extremal function of a functional. A function $u(x)$ in $\widetilde{L_{2}^{(m)}}(H)$ is called extremal for a given error functional $\ell(x)$ if the identity

$$
(\ell(x), u(x))=\left\|\ell \widetilde{L_{2}^{(m) *}}(H)\right\|\left\|\widetilde{L_{2}^{(m)}}(H)\right\|
$$

holds true. The space $\widetilde{L_{2}^{(m)}}(H)$ is the Hilbert one with the scalar product

$$
\langle\varphi, \psi\rangle_{m}=\int_{\Omega_{0}} \sum_{|\alpha|=m} \frac{m !}{\alpha !} D^{\alpha} \varphi(x) D^{\alpha} \psi(x) d x .
$$

By the Riesz theorem, each bounded functional $\ell(x)$ in the Hilbert space is represented as the scalar product

$$
(\ell, \varphi)=\left\langle\varphi, \psi_{\ell}\right\rangle_{m}
$$

for each $\varphi(x)$ in $\widetilde{L_{2}^{(m)}}(H)$. $\psi_{\ell}(x)$ is the function in $\widetilde{L_{2}^{(m)}}(H)$ determined uniquely by the functional $\ell(x)$ and extremal for it. Integrating by parts in the sense of generalized functions theory in the right hand side of (4) and employing the periodicity of the functions $\varphi(x)$ and $\psi_{\ell}(x)$, we obtain the identity

$$
(\ell, \varphi)=(-1)^{m} \int_{\Omega_{0}} \Delta^{m} \psi_{\ell}(x) \varphi(x) d x
$$

Thus, the function $\psi_{\ell}(x)$ is a generalized solution to the equation

$$
\Delta^{m} \psi_{\ell}(x)=(-1)^{m} \ell(x) .
$$

The following lemma holds true.

Lemma 1. An explicit expression for an extremal function to error functional (3) is given by the formula

$$
\psi_{l}(x)=(-1)^{m}\left[B_{2 m}(x-z)-\sum_{k=1}^{N} C_{k}(z) B_{2 m}\left(x-x^{(k)}\right)+d_{0}\right],
$$

where $d_{0}$ constant, the Bernoulli-Sobolev function $B_{2 m}(x)=(-1)^{m} \sum_{\gamma \neq 0} \frac{\exp \left(-2 \pi i H^{-1} x \gamma\right)}{\left|2 \pi H^{-1 *} \gamma\right|^{2 m}}$ is the periodic fundamental solution to the operator $\Delta^{m}$.

Proof. Let us find a periodic solution to equation (5). Applying the Fourier transform to both sides of (5) and employing the well-known formulas $F[\delta(x-z)]=e^{2 \pi i p^{*} z}, F\left[\phi_{0}\left(H^{-1}\right) x\right]=$ $\phi_{0}\left(H^{*} p\right)($ see $[9])$, we get

$$
\left[\sum_{j=1}^{n}\left(2 \pi i p_{j}\right)^{2}\right]^{m} F\left[\psi_{\ell}(x)\right]=(-1)^{m}\left[\left(e^{-2 \pi i p^{*} z}-\sum_{k=1}^{N} C_{k}(z) e^{2 \pi i p^{*} x^{(k)}}\right) \phi_{0}\left(H^{*} p\right)\right] .
$$

Here $p^{*}$ is the row vector $\left(p_{1}, p_{2}, \ldots, p_{n}\right)$ adjoint with the column-vector $p$. By (1) the right hand side in (7) vanishes in a vicinity of the origin. This is why we can divide both sides of (7) by $(2 \pi i)^{2 m}\left(\sum_{j=1}^{n} p_{j}^{2}\right)^{m}$. The function $F\left[\psi_{\ell}(x)\right]$ is determined by equation (7) up to an additive term

$$
(-1)^{m} d_{0} \delta(p)+\sum_{0<|\alpha| \leqslant 2 m} d_{\alpha} D^{\alpha} \delta(p)
$$


Since, however, $F\left[\psi_{\ell}(x)\right]$ must be a harrow-like, i.e., a linear combination of $\delta$-functions concentrated at the nodes of the integer lattice, all the terms except $(-1)^{m} d_{0} \delta(p)$ must be neglected.

Therefore,

$$
\begin{gathered}
F\left[\psi_{\ell}(x)\right]=(-1)^{m} d_{0} \delta(p)+\frac{\exp \left(2 \pi i p^{*} z\right) \phi_{0}\left(H^{*} p\right)}{(2 \pi)^{2 m}\left(\sum_{j=1}^{n} p_{j}^{2}\right)^{m}-} \\
-\frac{\sum_{k=1}^{N} C_{k}(z) \exp \left(2 \pi i p^{*} x^{(k)}\right) \phi_{0}\left(H^{*} p\right)}{(2 \pi)^{2 m}\left(\sum_{j=1}^{n} p_{j}^{2}\right)^{m}} .
\end{gathered}
$$

Replacing $\phi_{0}\left(H^{*} p\right)$ by the series in $\delta$-functions and applying the inverse Fourier transform to both sides of (8), we obtain (6) that proves the lemma.

\section{NORM OF ERROR FUNCTIONAL OF INTERPOLATION FORMULA}

The norm of the error functional of the interpolation formula is expressed via a bilinear form of the formula's coefficients and the value of the extremal function $\psi_{\ell}(x)$. Since the space $\widetilde{L_{2}^{(m)}}(H)$ is the Hilbert one, we have

$$
\left(\ell, \psi_{\ell}\right)=\left\|\ell \widetilde{L_{2}^{(m) *}}(H)\right\|\left\|\psi_{\ell} \mid \widetilde{L_{2}^{(m)}}(H)\right\|=\left\|\ell \widetilde{L_{2}^{(m) *}}(H)\right\|^{2} .
$$

Employing formulas (3), (6), (9), after straightforward calculations we get

$$
\begin{gathered}
\left\|\ell \mid \widetilde{L_{2}^{(m) *}}(H)\right\|^{2}=\int_{\Omega_{0}} \ell(x) \psi_{\ell}(x) d x= \\
=(-1)^{m} \int_{\Omega_{0}}\left(\delta(x-z)-\sum_{k=1}^{N} C_{k}(z) \delta\left(x-x^{(k)}\right)\right) * \phi_{0}\left(H^{-1} x\right)\left(B_{2 m}(x-z)-\right. \\
\left.-\sum_{k=1}^{N} C_{k}(z) B_{2 m}\left(x-x^{(k)}\right)+d_{0}\right) d x .
\end{gathered}
$$

By (1) and the definition of delta-function it yields

$$
\begin{gathered}
\left\|\ell \mid \widetilde{L_{2}^{(m) *}}(H)\right\|^{2}= \\
=(-1)^{m} \int_{\Omega_{0}}\left(\sum_{\beta} \delta(x-z-H \beta)-\sum_{k=1}^{N} C_{k}(z) \sum_{\beta} \delta\left(x-x^{(k)}-H \beta\right)\right) \times \\
\times\left(B_{2 m}(x-z)-\sum_{k=1}^{N} C_{k}(z) B_{2 m}\left(x-x^{(k)}\right)\right) d x .
\end{gathered}
$$

Employing the characteristic function $\chi_{\Omega_{0}}(x)$ of the domain $\Omega_{0}$, we rewrite the latter expression obtained from the norm of the functional as

$$
\begin{gathered}
\left\|\ell(x) \mid \widetilde{L_{2}^{(m) *}}(H)\right\|^{2}=(-1)^{m}\left(\sum_{\beta} \chi_{\Omega_{0}}(z+H \beta) B_{2 m}(H \beta)-\right. \\
-\sum_{k=1}^{N} C_{k}(z) \sum_{\beta} \chi_{\Omega_{0}}(z+H \beta) B_{2 m}\left(z+H \beta-x^{(k)}\right)-
\end{gathered}
$$




$$
\begin{gathered}
-\sum_{k=1}^{N} C_{k}(z) \sum_{\beta} \chi_{\Omega_{0}}\left(x^{(k)}+H \beta\right) B_{2 m}\left(x^{(k)}+H \beta-z\right)+ \\
\left.+\sum_{k=1}^{N} C_{k}(z) \sum_{k^{\prime}=1}^{N} C_{k^{\prime}}(z) \sum_{\beta} \chi_{\Omega_{0}}\left(x^{(k)}+H \beta\right) B_{2 m}\left(x^{(k)}+H \beta-x^{\left(k^{\prime}\right)}\right)\right) .
\end{gathered}
$$

Since $x^{(k)} \in \Omega_{0}, z \in \Omega_{0}, \sum_{\beta} \chi_{\Omega_{0}}(y+H \beta)=1, y \in \Omega_{0}$, employing the evenness of the BernoulliSobolev function $B_{2 m}(y)$, i.e., $B_{2 m}(y)=B_{2 m}(-y)$, we finally have

$$
\begin{gathered}
\left\|\ell(x) \mid \widetilde{L_{2}^{(m) *}}(H)\right\|^{2}= \\
=(-1)^{m}\left(B_{2 m}(0)-2 \sum_{k=1}^{N} C_{k}(z) B_{2 m}\left(z-x^{(k)}\right)+\sum_{k=1}^{N} C_{k}(z) \sum_{k^{\prime}=1}^{N} C_{k^{\prime}}(z) B_{2 m}\left(x^{(k)}-x^{\left(k^{\prime}\right)}\right)\right) .
\end{gathered}
$$

We note that the square of the norm of the functional $\ell$ is a nonnegative second order polynomial of the $N$ real variables $C_{1}(z), \ldots, C_{N}(z)$. This polynomial is considered on the linear manifold $\sum_{k=1}^{N} C_{k}(z)=1$. This function is obviously attains the minimum at a point $C^{0}(z)=\left(C_{1}^{0}(z), \ldots, C_{N}^{0}(z)\right)$. Owing to a strong convexity of the norm of the Hilbert space, this point is unique.

To find the point of minimum for norm (10) under the condition (1), one can apply the Lagrange method of undeterminate multipliers.

Consider an auxiliary function

$$
\Psi(C(z), \lambda)=\|\ell\|^{2}-2(-1)^{m} \lambda(\ell, 1) .
$$

Equating to zero the partial derivatives of $\Psi(C(z), \lambda)$ w.r.t. $C_{k}(z)$ and $\lambda$, we obtain the system of equations

$$
\begin{gathered}
\sum_{k=1}^{N} C_{k}^{0}(z) B_{2 m}\left(x^{\left(k^{\prime}\right)}-x^{(k)}\right)+\lambda^{0}=B_{2 m}\left(z-x^{\left(k^{\prime}\right)}\right), \quad k^{\prime}=1,2, \ldots, N, \\
\sum_{k=1}^{N} C_{k}^{0}(z)=1 .
\end{gathered}
$$

Consider the system on the grid, i.e., let $x^{(\gamma)}=H h \gamma$. We call such interpolation formula a grid one. Here $h$ is a small parameter which is the grid spacing. Then the system casts into the form

$$
\begin{gathered}
\sum_{h H \gamma \in \Omega_{0}} \stackrel{\circ}{C}_{h H \gamma}(z) B_{2 m}(h H(\beta-\gamma))+\lambda^{0}=B_{2 m}(z-h H \beta), \quad h H \beta \in \Omega_{0}, \\
\sum_{h H \gamma \in \Omega_{0}} \stackrel{\circ}{C}_{h H \gamma}(z)=1 .
\end{gathered}
$$

Employing the convolution of two functions of a discrete argument defined by the formula (see [9])

$$
f[\beta] * g[\beta]=\sum_{\gamma=-\infty}^{\infty} f[\gamma] \cdot g[\beta-\gamma],
$$

we write system (13), (14) as convolution equations,

$$
B_{2 m}[\beta] *\left(\stackrel{\circ}{C}([\beta] ; z) \chi_{\Omega_{0}}[\beta]\right)+\lambda^{0}=B_{2 m}(z-[\beta]), \quad[\beta] \in \Omega_{0},
$$




$$
\sum_{[\beta] \in \Omega_{0}} \stackrel{\circ}{C}([\beta] ; z)=1
$$

where $[\beta]=h H \beta$.

System of equations (15), 16) is called Wiener-Hopf system and now the problem is to solve this system w.r.t. $\stackrel{\circ}{C}([\beta] ; z)$ and $\lambda^{0}$.

Problem A. Find a function $\stackrel{\circ}{C}([\beta] ; z)$ and $\lambda$ satisfying the Wiener-Hopf system.

In solving Problem A an important role is played by certain properties of a discrete analogue $D_{h H}^{(m)}[\beta]$ of the polyharmonic operator $\Delta^{m}$; these properties will be proven in the next section.

Traditionally, a discrete analogue of the polyharmonic operator appears as the result of the change of the derivatives involved in $\Delta^{m}$ by appropriate finite differences. Another idea of discretization of a differential operator coming from S.L. Sobolev [9] reads as follows. In the equation determining the fundamental solution (in our case it is $\Delta^{m} B_{2 m}(x)=\Phi_{0}(x)$ in the periodic version) one passes to the discrete arguments of the fundamental solution and $\delta$-function replacing the polyharmonic operator by some function of discrete variables acting as the discrete convolution on the discretized fundamental solution.

It is given by the formula

$$
D_{h H}^{m}[\beta] * B_{2 m}[\beta]=\sum_{\gamma} \delta[\beta-\gamma / h]
$$

where $[\beta]=h H \beta, \beta \in \mathbb{Z}^{n}, 1 / h$ is a natural number, $\delta[\beta] \equiv \delta_{0}^{|\beta|}$ is the Kronecker delta.

The operator of discrete convolution defined by this formula $D_{h H}^{m}[\beta] *$ is a discrete analogue to the polyharmonic operator.

\section{NeW Properties of operator $D_{h H}^{(m)}[\beta]$}

Let us justify new properties of the convolution operator $D_{h H}^{(m)}[\beta]$. We note that as $n=1$, a discrete analogue to the differential operator $d^{2 m} / d x^{2 m}$ was constructed in work [10].

We find the function of discrete variable $D_{h H}^{(m)}[\beta]$ satisfying the identity

$$
h^{n} D_{h H}^{(m)}[\beta] * B_{2 m}[\beta]=\Phi[\beta]-h^{n} .
$$

Here $B_{2 m}[\beta]$ is the Bernoulli-Sobolev function defined by the formula

$$
B_{2 m}[\beta]=(-1)^{m} \sum_{\gamma \neq 0} \frac{\exp \left(-2 \pi i \gamma^{*} h \beta\right)}{\left|2 \pi H^{-1 *} \gamma\right|^{2 m}},
$$

and $\Phi[\beta]$ is a discrete periodic delta-function and

$$
\Phi[\beta]=\sum_{\gamma} \delta\left[\beta-h^{-1} \gamma\right],
$$

$[\beta]=h H \beta, h^{-1}$ is a natural number, $\delta\left[\beta-h^{-1} \gamma\right]$ is the discrete delta-function defined by the formula

$$
\delta\left[\beta-h^{-1} \gamma\right]=\left\{\begin{array}{l}
1, \text { if } \beta-h^{-1} \gamma=0 \\
0, \text { if } \beta-h^{-1} \gamma \neq 0,
\end{array}\right.
$$

$\beta=\uparrow\left(\beta_{1}, \beta_{2}, \ldots, \beta_{n}\right)$ is the column vector, $\beta^{*}=\left(\beta_{1}, \beta_{2}, \ldots, \beta_{n}\right)$ is the row vector, $\beta_{j} \in \mathbb{Z}, \mathbb{Z}$ is the set of integers. 
Lemma 2. A solution to convolution equation (17) is determined by the formula

$$
D_{h H}^{(m)}[\beta]=\frac{1}{\left|\Omega_{1}\right|} \int_{\Omega_{1}} \Gamma_{h H}^{(m)}(p) \exp \left(2 \pi i \beta^{*} h H^{*} p\right) d p,
$$

where $\Omega_{1}$ is the fundamental parallelepiped for the matrix $h^{-1} H^{-1 *},\left|\Omega_{1}\right|$ is the volume of the domain $\Omega_{1}$,

$$
\Gamma_{h H}^{(m)}(p)=\left[\frac{(-1)^{m}}{(2 \pi)^{2 m}} \sum_{\gamma} \frac{1}{\left|p-h^{-1} H^{-1 *} \gamma\right|^{2 m}}\right]^{-1}, \quad p \neq h^{-1} H^{-1 *} \gamma
$$

Proof. To find $D_{h H}^{(m)}[\beta]$, we employ the Fourier transformation. The class of discrete functions and the class of harrow-like functions are known to be isomorphic (see [9]). Employing this fact, we pass from the discrete functions to harrow-like ones

$$
\breve{\psi}(x)=\sum_{\beta} h^{n} \psi[\beta] \delta(x-h H \beta) .
$$

For $\Phi[\beta]$ defined by formula $(18)$ we have

$$
\vec{\Phi}(x)=\sum_{\beta} h^{n} \Phi[\beta] \delta(x-h H \beta)=h^{n} \sum_{\beta} \sum_{\gamma} \delta\left[\beta-h^{-1} \gamma\right] \delta(x-h H \beta) .
$$

By (19) it implies

$$
\vec{\Phi}(x)=\sum_{\gamma} h^{n} \delta(x-H \gamma)=h^{n} \phi_{0}\left(H^{-1} x\right) .
$$

Equation (17) on the class of harrow-like function becomes

$$
h^{n} \widetilde{D}_{h H}^{(m)}(x) * \widetilde{B}_{2 m}(x)=h^{n} \phi_{0}\left(H^{-1} x\right)-h^{n} \phi_{0}\left(h^{-1} H^{-1} x\right),
$$

where $\phi_{0}\left(h^{-1} H^{-1} x\right)=\sum_{\beta} h^{n} \delta(x-h H \beta)$.

As is known, the Fourier transform of the functions $\phi_{0}\left(H^{-1} x\right)$ and $\phi_{0}\left(h^{-1} H^{-1} x\right)$ is provided by the formulas

$$
\begin{gathered}
F\left[\phi_{0}\left(H^{-1} x\right)\right]=\int e^{2 \pi i p^{*} x} \phi_{0}\left(H^{-1} x\right) d x= \\
=\int e^{2 \pi i p^{*} x} \sum_{\beta} \delta(x-H \beta) d x=\sum_{\beta} \int e^{2 \pi i p^{*} x} \delta(x-H \beta) d x=\sum_{\beta} e^{2 \pi i p^{*} H \beta}, \\
F\left[\phi_{0}\left(h^{-1} H^{-1} x\right)\right]=\int e^{2 \pi i p^{*} x} \phi_{0}\left(h^{-1} H^{-1} x\right) d x=\sum_{\beta} h^{n} e^{2 \pi i p^{*} h H \beta} .
\end{gathered}
$$

Employing the well-known Poisson formula

$$
h^{n} \sum_{\beta} e^{2 \pi i p^{*} h H \beta}=\sum_{\beta} \delta\left(p-h^{-1} H^{-1 *} \beta\right),
$$

we rewrite identities (23) and (24) as

$$
\begin{gathered}
F\left[\phi_{0}\left(H^{-1} x\right)\right]=\sum_{\beta} \delta\left(p-H^{-1 *} \beta\right), \\
F\left[\phi_{0}\left(h^{-1} H^{-1} x\right)\right]=\sum_{\beta} \delta\left(p-h^{-1} H^{-1 *} \beta\right) .
\end{gathered}
$$


Let us calculate the Fourier transform of the function

$$
\overparen{B}_{2 m}(x)=h^{n}(-1)^{m} \sum_{\beta} \sum_{\gamma \neq 0} \frac{\exp \left(-2 \pi i \gamma^{*} h \beta\right)}{\left|2 \pi H^{-1 *} \gamma\right|^{2 m}} \delta(x-h H \beta) .
$$

By the definition of the Fourier transform we get

$$
\begin{gathered}
F\left[\breve{B}_{2 m}(x)\right]=\int e^{2 \pi i p^{*} x} \breve{B}_{2 m}(x) d x= \\
=\frac{(-1)^{m}}{(2 \pi)^{2 m}} \sum_{\beta} h^{n} \sum_{\gamma \neq 0} \frac{\exp \left(-2 \pi i \gamma^{*} h \beta\right) \exp \left(2 \pi i p^{*} h H \beta\right)}{\left|H^{-1 *} \gamma\right|^{2 m}}= \\
=\frac{(-1)^{m}}{(2 \pi)^{2 m}} \sum_{\beta} h^{n} \sum_{\gamma \neq 0} \frac{\exp \left(2 \pi i\left(p^{*} H-\gamma^{*}\right) h \beta\right)}{\left|H^{-1 *} \gamma\right|^{2 m}} .
\end{gathered}
$$

By (25) we have

$$
\begin{gathered}
h^{n} \sum_{\beta} \exp \left(2 \pi i\left(p^{*} H-\gamma^{*}\right) h \beta\right)=\sum_{\beta} h^{n} \exp \left(2 \pi i\left(p^{*}-\gamma^{*} H^{-1}\right) h H \beta\right)= \\
=\sum_{\beta} \delta\left(p-H^{-1 *} \gamma-h^{-1} H^{-1 *} \beta\right) .
\end{gathered}
$$

Then

$$
F\left[\overleftarrow{B}_{2 m}(x)\right]=\frac{(-1)^{m}}{(2 \pi)^{2 m}} \sum_{\beta} \sum_{\gamma \neq 0} \frac{\delta\left(p-H^{-1 *}\left(\gamma+h^{-1} \beta\right)\right)}{\left|H^{-1 *} \gamma\right|^{2 m}} .
$$

Making the change of the variables $\gamma+h^{-1} \beta=k$, we finally get

$$
F\left[\overleftarrow{B}_{2 m}(x)\right]=\frac{(-1)^{m}}{(2 \pi)^{2 m}} \sum_{\beta} \sum_{\substack{k \\ k h \notin \mathbb{Z}}} \frac{\delta\left(p-H^{-1 *} k\right)}{\left|H^{-1 *}\left(k-h^{-1} \beta\right)\right|^{2 m}}
$$

Applying the Fourier transform to both sides of (22), using formulas (26), (27), and (28), and dividing by $h^{n}>0$, we obtain the following equation,

$$
F\left[\overleftrightarrow{D}_{h H}^{(m)}(x)\right] \cdot \frac{(-1)^{m}}{(2 \pi)^{2 m}} \sum_{\beta} \sum_{\substack{\gamma \\ \gamma h \notin \mathbb{Z}}} \frac{\delta\left(p-H^{-1 *} \gamma\right)}{\left|H^{-1 *}\left(\gamma-h^{-1} \beta\right)\right|^{2 m}}=\sum_{\substack{\gamma \\ h \gamma \notin \mathbb{Z}}} \delta\left(p-H^{-1 *} \gamma\right)
$$

It is known that

$$
\sum_{\beta} \sum_{\substack{\gamma \\ \gamma h \notin \mathbb{Z}}} \frac{\delta\left(p-H^{-1 *} \gamma\right)}{\left|H^{-1 *}\left(\gamma-h^{-1} \beta\right)\right|^{2 m}}=\sum_{\beta} \sum_{\substack{\gamma \\ \gamma h \notin \mathbb{Z}}} \frac{\delta\left(p-H^{-1 *} \gamma\right)}{\left|p-h^{-1} H^{-1 *} \beta\right|^{2 m}} .
$$

By (30), equation 29 is equivalent to

$$
F\left[\overleftarrow{D}_{h H}^{(m)}(x)\right] \cdot\left(\Gamma_{h H}^{(m)}(p)\right)^{-1}=1, \quad p \neq h^{-1} H^{-1 *} \gamma
$$

where $\Gamma_{h H}^{(m)}(p)$ is determined by formula (21). The function $\Gamma_{h H}^{(m)}(p)$ is periodic in $p$ with the periods matrix $h^{-1} H^{-1 *}$, real, and analytic for all $p \neq h^{-1} H^{-1 *} \gamma$.

By (31) we have

$$
F\left[\overparen{D}_{h H}^{(m)}(x)\right]=\Gamma_{h H}^{(m)}(p) .
$$

Applying the inverse Fourier transform to both sides of 32 and passing from harrow-like generalized functions to discrete ones, we arrive at (20) that proves the lemma. 
Lemma 3. The operator $D_{h H}^{(m)}[\beta]$ and the function $\exp \left(2 \pi i \sigma^{*} h H \beta\right)$ are related by the identity

$$
\begin{gathered}
D_{h H}^{(m)}[\beta] * \exp \left(2 \pi i \sigma^{*} h H \beta\right)= \\
=(-1)^{m}(2 \pi)^{2 m} \exp \left(2 \pi i \sigma^{*} h H \beta\right)\left[\sum_{\substack{\gamma \\
\gamma \neq h H^{*} \sigma}} \frac{1}{\left|h^{-1} H^{-1 *} \gamma-\sigma\right|^{2 m}}\right]^{-1} .
\end{gathered}
$$

Here $D_{h H}^{(m)}[\beta]$ is determined by formula (20).

Proof. We denote by $\overparen{T}(x)$ the convolution of harrow-like functions $\overleftrightarrow{D}_{h H}^{(m)}(x)$ and $\overparen{\exp }\left(2 \pi i \sigma^{*} x\right)$,

$$
\overparen{T}(x)=\overleftrightarrow{D}_{h H}^{(m)}(x) * \overparen{\exp }\left(2 \pi i \sigma^{*} x\right)
$$

Employing formula (32), we find the Fourier transform of the function $\vec{T}(x)$,

$$
\begin{gathered}
F[\overleftrightarrow{T}(x)]=F\left[\overleftrightarrow{D}_{h H}^{(m)}(x) * \overleftrightarrow{\exp }\left(2 \pi i \sigma^{*} x\right)\right]= \\
=F\left[\overleftarrow{D}_{h H}^{(m)}(x)\right] \cdot F\left[\overleftarrow{\exp }\left(2 \pi i \sigma^{*} x\right)\right]=\Gamma_{h H}^{(m)}(p) \cdot F\left[\overleftarrow{\exp }\left(2 \pi i \sigma^{*} x\right)\right]
\end{gathered}
$$

By the definition of harrow-like function and the Fourier transform of $\delta(x-h H \beta)$ we have

$$
\begin{gathered}
F\left[\overleftarrow{\exp }\left(2 \pi i \sigma^{*} x\right)\right]=F\left[\sum_{\beta} h^{n} \exp \left(2 \pi i \sigma^{*} h H \beta\right) \delta(x-h H \beta)\right]= \\
=\sum_{\beta} h^{n} \exp \left(2 \pi i \sigma^{*} h H \beta\right) \exp \left(2 \pi i p^{*} h H \beta\right)=\sum_{\beta} h^{n} \exp \left(2 \pi i\left(\sigma^{*}+p^{*}\right) h H \beta\right) .
\end{gathered}
$$

By (25) it yields

$$
F\left[\overparen{\exp }\left(2 \pi i \sigma^{*} x\right)\right]=\sum_{\beta} \delta\left(\sigma+p-h^{-1} H^{-1 *} \beta\right) .
$$

By formulas (21), (33) we get

$$
\begin{gathered}
F[\vec{T}(x)]=\left[\frac{(-1)^{m}}{(2 \pi)^{2 m}} \sum_{\gamma} \frac{1}{\left|p-h^{-1} H^{-1 *} \gamma\right|^{2 m}}\right]^{-1} \cdot \sum_{\beta} \delta\left(\sigma+p-h^{-1} H^{-1 *} \beta\right)= \\
=\sum_{\beta} \delta\left(\sigma+p-h^{-1} H^{-1 *} \beta\right)\left[\frac{(-1)^{m}}{(2 \pi)^{2 m}} \sum_{\gamma} \frac{1}{\left|h^{-1} H^{-1 *} \beta-\sigma-h^{-1} H^{-1 *} \gamma\right|^{2 m}}\right]^{-1}= \\
=\sum_{\beta} \delta\left(\sigma+p-h^{-1} H^{-1 *} \beta\right)\left[\frac{(-1)^{m}}{(2 \pi)^{2 m}} \sum_{\gamma} \frac{1}{\left|h^{-1} H^{-1 *}(\beta-\gamma)-\sigma\right|^{2 m}}\right]^{-1}, \\
p \neq h^{-1} H^{-1 *} \gamma, \quad \beta-\gamma \neq h H^{*} \gamma .
\end{gathered}
$$

Employing formula (25), we calculate the inverse Fourier transform of the function $\sum_{\beta} \delta\left(\sigma+p-h^{-1} H^{-1 *} \beta\right)$, and keeping in mind the definition of harrow-like functions, we get

$$
\begin{gathered}
F^{-1}\left[\sum_{\beta} \delta\left(\sigma+p-h^{-1} H^{-1 *} \beta\right)\right]=\sum_{\beta} \int e^{-2 \pi i x^{*} p} \delta\left(\sigma+p-h^{-1} H^{-1 *} \beta\right) d p= \\
=e^{2 \pi i x^{*} \sigma} \sum_{\beta} e^{-2 \pi i x^{*} h^{-1} H^{-1 *} \beta}=e^{2 \pi i x^{*} \sigma} h^{n} \sum_{\beta} \delta(x-h H \beta)=e^{2 \pi i \sigma^{*} x} \sum_{\beta} h^{n} \delta(x-h H \beta)= \\
=\sum_{\beta} h^{n} e^{2 \pi i \sigma^{*} h H \beta} \delta(x-h H \beta)=\overparen{\exp }\left(2 \pi i \sigma^{*} x\right) .
\end{gathered}
$$


Now, applying the inverse Fourier transform to both sides of formula (34) and taking into consideration (35), we obtain

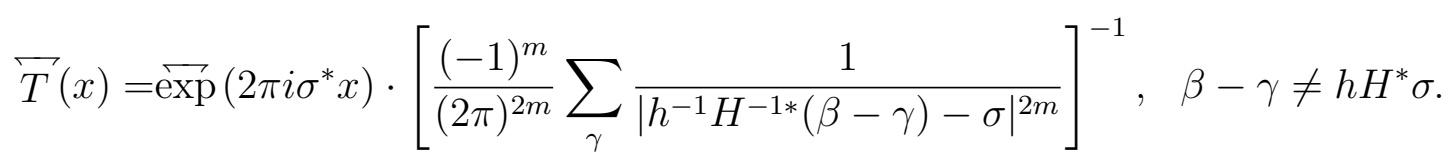

Passing from harrow-like functions to usual discrete functions, we have

$$
T[\beta]=\exp \left(2 \pi i \sigma^{*} h H \beta\right) \cdot\left[\frac{(-1)^{m}}{(2 \pi)^{2 m}} \sum_{\gamma} \frac{1}{\left|h^{-1} H^{-1 *}(\beta-\gamma)-\sigma\right|^{2 m}}\right]^{-1}, \beta-\gamma \neq h H^{*} \sigma .
$$

The proof is complete.

\section{Solution to Problem A}

The following lemma holds true.

Lemma 4. Let $g[\beta]$ be a discrete periodic function, i.e., $g[\beta]=g(h H \beta)=g(h H \beta+H \gamma)$, then the identity

holds true.

$$
g[\beta]=\left(g[\beta] \chi_{\Omega_{0}}[\beta]\right) * \Phi[\beta]
$$

Proof. Employing formulas (18), 19,

$$
\sum_{\gamma} \chi_{\Omega_{0}}(h H \beta+H \gamma)=1
$$

and the periodicity of the discrete function $g[\beta]$, we have

$$
\begin{gathered}
g[\beta]=g[\beta] \sum_{\gamma} \chi_{\Omega_{0}}(h H \beta+H \gamma)=\sum_{\gamma} g[\beta] \chi_{\Omega_{0}}(h H \beta+H \gamma)= \\
=\sum_{\gamma} g(h H \beta+H \gamma) \chi_{\Omega_{0}}(h H \beta+H \gamma)=\sum_{\gamma} g(h H \beta-H \gamma) \chi_{\Omega_{0}}(h H \beta-H \gamma)= \\
=\sum_{\gamma} g\left[\beta-h^{-1} \gamma\right] \chi_{\Omega_{0}}\left[\beta-h^{-1} \gamma\right]=\sum_{\gamma} \sum_{k} g[k] \chi_{\Omega_{0}}[k] \delta\left[\beta-k-h^{-1} \gamma\right]= \\
=\sum_{k} g[k] \chi_{\Omega_{0}}[k] \sum_{\gamma} \delta\left[\beta-k-h^{-1} \gamma\right]=\sum_{k} g[k] \chi_{\Omega_{0}}[k] \Phi[\beta-k]= \\
=\left(g[\beta] \chi_{\Omega_{0}}[\beta]\right) * \Phi[\beta] .
\end{gathered}
$$

The proof is complete.

Proof of Theorem. We employ the following well-known formula from [9],

$$
D_{h H}^{(m)}[\beta] *[\beta]^{k}=0 \text { as } k<2 m .
$$

Applying the operator $h^{n} D_{h H}^{(m)}[\beta]$ to both sides of equation (15), we obtain

$$
h^{n} D_{h H}^{(m)}[\beta] *\left(B_{2 m}[\beta] *\left(\stackrel{\circ}{C}([\beta] ; z) \chi_{\Omega_{0}}[\beta]\right)+\lambda\right)=h^{n} \cdot D_{h H}^{(m)}[\beta] * B_{2 m}(z-h H \beta), \quad h H \beta \in \Omega_{0} .
$$

Employing formulas (17), (36), (37), due to (38) we have

$$
\stackrel{\circ}{C}([\beta] ; z)-h^{n} \sum_{h H \beta \in \Omega_{0}} \stackrel{\circ}{C}([\beta] ; z)=h^{n} D_{h H}^{(m)}[\beta] * B_{2 m}(z-h H \beta), \quad h H \beta \in \Omega_{0} .
$$

By (16) we find

$$
\stackrel{\circ}{C}([\beta] ; z)=h^{n}+h^{n} \cdot D_{h H}^{(m)}[\beta] * B_{2 m}(z-h H \beta), \quad h H \beta \in \Omega_{0} .
$$


Since the function $B_{2 m}(z-h H \beta)$ is the Bernoulli-Sobolev one, it yields

$$
\begin{aligned}
& \stackrel{\circ}{C}([\beta] ; z)=h^{n}+h^{n} \cdot D_{h H}^{(m)}[\beta] *(-1)^{m} \sum_{\gamma \neq 0} \frac{\exp \left(-2 \pi i H^{-1}(z-h H \beta) \gamma\right)}{\left|2 \pi H^{-1 *} \gamma\right|^{2 m}}= \\
& \quad=h^{n}+(-1)^{m} h^{n} \sum_{\gamma \neq 0} \frac{\exp \left(-2 \pi i H^{-1} z \gamma\right)}{\left|2 \pi H^{-1 *} \gamma\right|^{2 m}} D_{h H}^{(m)}[\beta] * \exp \left(2 \pi i h \beta^{*} \gamma\right)= \\
& =h^{n}+(-1)^{m} h^{n} \sum_{\gamma \neq 0} \frac{\exp \left(-2 \pi i H^{-1} z \gamma\right)}{\left|2 \pi H^{-1 *} \gamma\right|^{2 m}} D_{h H}^{(m)}[\beta] * \exp \left(2 \pi i \gamma^{*} H^{-1}(h H \beta)\right) .
\end{aligned}
$$

Employing now Lemma 6, by (39) we obtain

$$
\stackrel{\circ}{C}([\beta] ; z)=h^{n}+(-1)^{m} h^{n} \sum_{\gamma \neq 0} \frac{\exp \left(-2 \pi i H^{-1} z \gamma\right)}{\left|2 \pi H^{-1 *} \gamma\right|^{2 m}}(-1)^{m}(2 \pi)^{2 m} \exp \left(2 \pi i \gamma^{*} H^{-1} h H \beta\right) \times
$$

$$
\times\left[\sum_{\substack{t \\ t \neq h \gamma}} \frac{1}{\left|h^{-1} H^{-1 *} t-H^{-1 *} \gamma\right|^{2 m}}\right]^{-1} .
$$

Introducing the notation $K(\gamma)=\left[\sum_{\substack{t \\ t \neq h \gamma}} \frac{1}{\left|H^{-1 *}\left(h^{-1} t-\gamma\right)\right|^{2 m}}\right]^{-1}$, we obtain the final form of the optimal coefficients,

$$
\stackrel{\circ}{C}([\beta] ; z)=h^{n}\left(1+\sum_{\gamma \neq 0} \frac{\exp \left(2 \pi i H^{-1}\left(H h \beta^{*}-z\right) \gamma\right)}{\left|H^{-1 *} \gamma\right|^{2 m}} \cdot K(\gamma)\right) .
$$

The proof is complete.

Acknowledgments. We are sincerely grateful to professor M.D. Ramazanov for the discussion of the results of the present work.

\section{BIBLIOGRAPHY}

1. S.L. Sobolev. On the interpolation of functions of $n$ variables. // Dokl. AN SSSR. 1961. V. 137, No. 4, P. 778-781. [Sov. Math., Dokl. 1961. V. 2. P. 343-346.]

2. J.C. Holladay. Smoothest curve approximation. // Math. Tables Aids Comput. 1957. V. 11, No. 60. P. 223-243.

3. J.H. Ahlberg, E.N. Nilson, J.L. Walsh. The theory of splines and their applications. Academic Press, N.Y. 1967.

4. S.B. Stechkin, Yu.N. Subbotin. Splines in numerical mathematics. Nauka, Moscow. 1976. (in Russian).

5. P.J. Laurent. Approximation et optimisation. Hermann Blume, Paris. 1972.

6. V.A. Vasilenko. Spline-functions: theory, algorithms, programs. Nauka, Novosibirsk, 1984. (in Russian).

7. M.I. Ignatov, A.B. Pevnyj. Natural splines of many variable, Nauka, Leningrad. 1991

8. N.P. Korenichuk. Exact constants in approximation theory. Nauka, Moscow. 1981. [Cambridge University Press, Cambridge, 1991.]

9. S.L. Sobolev. The theory of cubature formulas. Nauka, Moscow. 1974. [Kluwer Academic Publishers, Dordrecht, 1996.] 
10. H.M. Shadimetov. Discrete analogue of operator $d^{2 m} / d x^{2 m}$ and its construction // Voprosy vychislit. i prikl. matem. Tashkent. 1985. No. 79. P. 22-35. (in Russian).

Nilufar Husenovna Mamatova,

Institute of Mathematics, National University of Uzbekistan, Do'rmon yo'li str. 29, 100125, Tashkent, Uzbekistan

Abdullo Rakhmonovich Hayotov,

Institute of Mathematics, National University of Uzbekistan, Do'rmon yo'li str. 29,

100125, Tashkent, Uzbekistan

E-mail: hayotov@mail.ru

Kholmatvoj Makhkambaevich Shadimetov,

Institute of Mathematics, National University of Uzbekistan, Do'rmon yo'li str. 29,

100125, Tashkent, Uzbekistan 